\title{
Die sowjetischen Massenmedien. Geschichte, Funktion, Struktur
}

\author{
von Paul Roth
}

\section{Geschichte der Massenmedien}

In Rußland war die Presse eine Schöpfung des Staates. Sie wurde bestimmt von der Politik der Zaren. Zensur wurde ausgeuibt, Verbote wurden verhängt. Nach 1865 wurde die Zensur gemildert. 1905 versprach der Zar Freiheit des Gewissens und der Rede, doch wurde keine volle Pressefreiheit gewährt. Die Entwicklung der Massenmedien in Rußland, der UdSSR, wird mitbestimmt durch die Größe des Landes, das elf Zeitzonen umfaßt. Ferner leben auf dem Territorium mehr als 100 Nationalitäten und Völkerschaften.

Die Entwicklung der Massenmedien ab 1917 wird geprägt durch die Funktionsbestimmung der Massenmedien, die auf Lenin zurückgeht. Lenin forderte (1900), daß die Zeitung ,nicht nur ein kollektiver Propagandist und kollektiver Agitator, sondern auch ein kollektiver Organisator" sein müsse. Die Informationsfunktion trat in den Hintergrund. Information ist - nach Lenin - „Agitation durch Fakten“. Die sogenannte bürgerliche Presse war in den Augen Lenins eine Säule der Ausbeuterordnung. Diese leninschen Funktionsbestimmungen wurden später auf alle Massenmedien übertragen.

Im November 1917 leitete das „Dekret über die Presse“ die Vernichtung der nichtbolschewistischen Presse ein. Die Druckereien wurden beschlagnahmt, das Anzeigenwesen wurde verstaatlicht. 1920 wurde im ZK eine Agit-Prop-Abteilung zur Lenkung der Medien geschaffen. Die Nachrichtenagentur ROSTA war zugleich Zentralstelle für Propaganda und Agitation. 1921 lag das Informations- und Meinungsmonopol in der Hand der Machthaber. Eine planvolle Entwicklung der Presse war im Kriegskommunismus (19.17-1921) nicht möglich. Der blutige Bürgerkrieg, der Mangel an Papier und Maschinen, die große Zahl von Analphabeten, der Zusammenbruch des Wirtschaftskreislaufs führten dazu, daß 1921 die Periodika kostenlos verteilt wurden. In der „Neuen Ökonomischen Politik“ (1921-1928) wurden die Periodika wieder verkauft. Die Zeitungspresse schrumpfte. Die Zeitschriftenpresse wuchs, nachdem Privatverlage wieder Zeitschriften herausbringen durften. Als eigene Zensurbehörde wurde GLAWLIT (1922) geschaffen, sie besteht bis heute (trägt inzwischen allerdings eine andere Bezeichnung). Die Forderung nach Pressefreiheit für alle politischen Gruppen wurde ein für allemal von Lenin und Stalin zurückgewiesen. Noch vor Beendigung der „Neuen OOkonomischen Politik“ waren die Privatverlage verschwunden.

Die Nachrichtenagentur TASS trat an die Stelle von ROSTA (1925). Das Zeitungsnetz konnte vergrößert werden, neue Zeitungen in den Sprachen der Völker wurden geschaffen. Die Zeitungspresse wurde entsprechend der territorialen Gliederung der UdSSR gegliedert, gleichzeitig nach Lesergruppen differenziert. Der Rundfunk steckte noch in den Kinderschuhen. Die gesamte Medienentwicklung vollzog sich unter Aufsicht und Lenkung durch die Partei.

Die Jahre 1928-1941 waren gekennzeichnet durch den Personenkult Stalins, der die Lenkung aller Massenmedien an sich zog. Presse und Rundfunk erhielten eine pyramidenartige Hierarchie, an deren Spitze die „Prawda“ trat. Die Zeitungspresse wuchs, die Zeitschriftenpresse nahm ab, nachdem die Privatverlage verschwunden waren. Die

Dr. Paul Roth ist Professor für Politikwissenschaft und Publizistik an der Hochschule der Bundeswehr in München und Lehrbeauftragter an der Universität München. Anerkannter Experte zur Medienpolitik der Sowjetunion. 
zentral betriebene Ausweitung des Rundfunks sollte vor allem die ländlichen Gebiete erreichen. 1939 waren knapp 19 Prozent der Bevölkerung im Alter von über 9 Jahren Analphabeten. Der Rundfunk blieb jedoch überwiegend auf Großstädte beschränkt. Die meisten Empfänger waren Drahtfunkempfänger, die auf ein bestimmtes Programm eingestellt waren.

Während des „Großen Vaterländischen Krieges“(1941-1945) wurde ein Teil der Rundfunkstationen vernichtet, die Anzahl und Auflage der Zeitungen und Zeitschriften sank gewaltig. Die Lenkung von Propaganda, Agitation, der Massenmedien, der Nachrichtengebung wurde vom 1941 geschaffenen SOWINFORMBÜRO vorgenommen.

In den Nachkriegsjahren unter Stalin (1945-1953) blieb die Presse das wichtigste Massenmedium. Die Zeitungspresse konnte 1951 den Vorkriegsstand erreichen. Die Allunionsorgane (also die leitenden Zeitungen) konnten mehr als ein Drittel der Jahresgesamtauflage für sich beanspruchen. Gewaltig gesteigert wurde die Anzahl der Rundfunkstationen. Dreiviertel der Empfänger waren Drahtfunkempfänger. Das Fernsehen befand sich im Aufbau. Eine Kette von ZK-Beschlüssen sorgte für Lenkung und Orientierung der Massenmedien. Chruschtschow (1954-1964) bemühte sich darum, die Massenmedien zu modernisieren, ohne die Lenkung durch die Partei zu schwächen. 1957 wurde das Staat liche Komitee für Rundfunk und Fernsehen geschaffen, 1963 das Staatliche Komitee für das Druckwesen. 1959 wurde der sowjetische Journalistenverband gegründet. Das SOWINFORMBÜRO, das nach dem Kriegsende Auslandspropaganda betrieben hatte, wurde durch die 1961 neugeschaffene "gesellschaftliche" Agentur NOVOSTI $(A P N)$ ersetzt. TASS stieg zur Weltagentur auf. Die Förderung des ,individuellen Abonnements“, die Verbesserung der Aufmachung, trug mit dazu bei, daß die Zeitungsauflagen gewaltig anstiegen. Die Gesamtzahl sank, da die Rayonzeitungen eingestellt wurden. Das Zeitschriftenwesen fächerte sich weit auf. Die Anzahl der Rundfunkstationen und der Empfänger stieg gewaltig. 1960 begann Moskau damit, sein Programm ganztags auszustrahlen. Die Anzahl der Fernsehzentren (sie produzieren Programme) wuchs anfangs recht unkoordiniert. Das Moskauer Fernsehen begann 1955 mit regelmäßigen Sendungen ohne freie Tage, 1956 folgte das zweite Moskauer Programm. Die Partei übernahm auch für das Fernsehen Anleitung und Kontrolle.

In der Ära Breschnew (1964-1982) wuchsen Zeitungs- und Zeitschriftenauflagen weiter kontinuierlich. Durch Ausweitung des Kabelnetzes und mit Hilfe von drahtloser Ubertragung wurde die UdSSR voll "radiofiziert", wobei das Moskauer Programm überall den Kern der Sendungen bildet. Die Anzahl der Fernseh-Programmzentren wuchs auf 134 (1969), um dann wieder abzunehmen. Rund 86 Prozent der Bevölkerung können fernsehen. Kabellinien und Satelliten sorgen für die Übertragung der Moskauer Programme. Die Staatsagentur TASS wurde 1971 zum Staatskomitee angehoben.

Die sowjetischen Zahlenangaben über die Massenmedien sind bis zum Ende der dreißiger Jahre vielfach widersprüchlich, bis in die fünfziger Jahre hinein ungenau und lückenhaft. Seitdem sind die Angaben auch nicht immer vollständig. Die Angaben über Rundfunk-und Fernsehempfänger sind Schätzungen, da keine Abonnementsgebühren gezahlt werden. Nur ein Teil der Rundfunkstationen produziert selber Programme. Die folgenden Tabellen stützen sich - unter Korrektur eindeutiger Fehler - vor allem auf die drei Ausgaben der „Großen Sowjet-Enzyklopädie“ und die Jahresbände „Pẻcat' SSSR v. 19.. godu“ (siehe Literaturverzeichnis). 
Entwicklung der Zeitungspresse

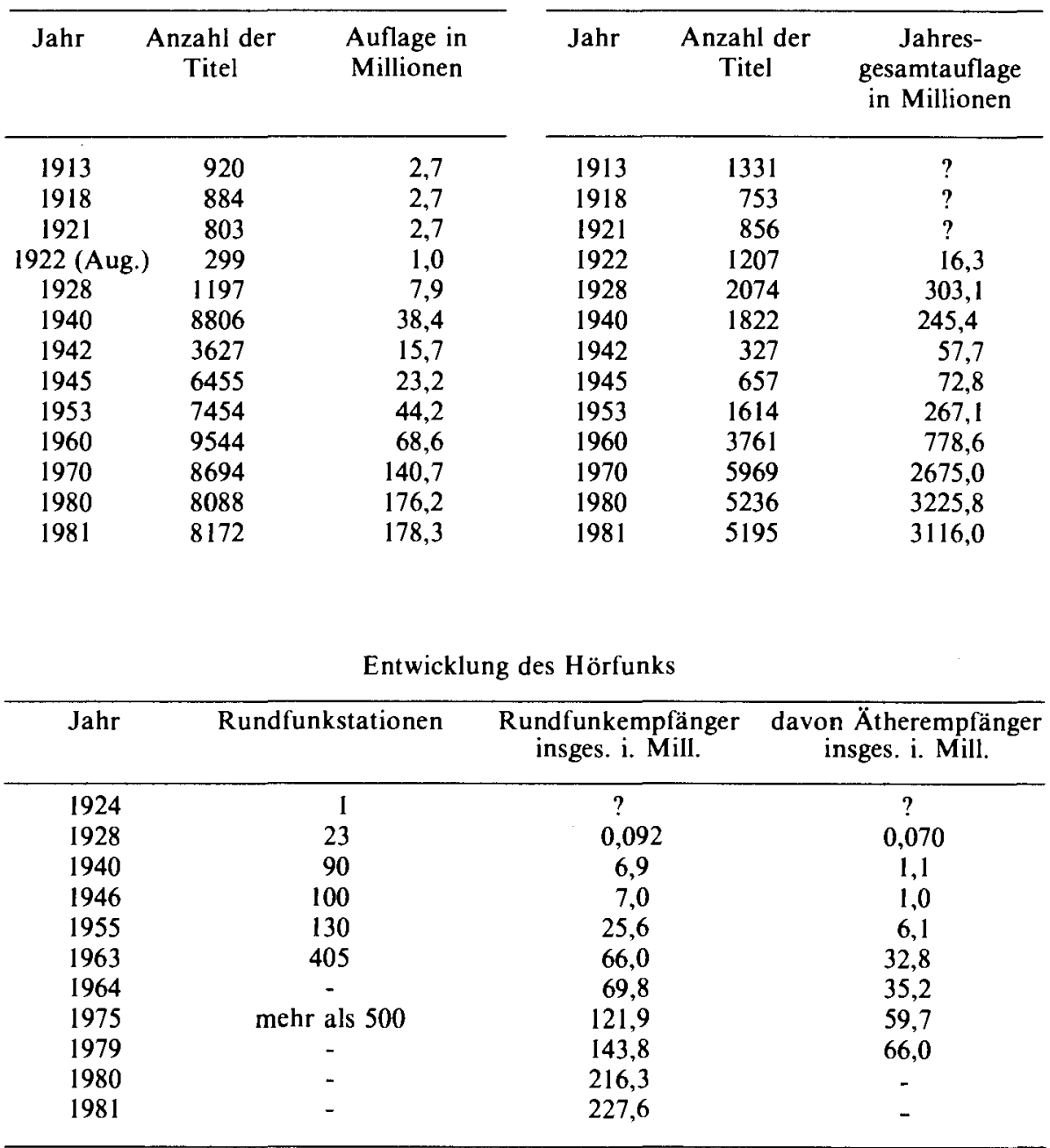

Entwicklung der Zeitschriftenpresse 


\begin{tabular}{cccc}
\hline Jahr & $\begin{array}{c}\text { Fernsehzentren } \\
\text { (Programmzentren) }\end{array}$ & $\begin{array}{c}\text { Fernsehzentren und } \\
\text { Relaisstationen zus. }\end{array}$ & $\begin{array}{c}\text { Benutzte Empfänger } \\
\text { in Millionen }\end{array}$ \\
\hline 1940 & 3 & $?$ & $?$ \\
1945 & 1 & $?$ & $?$ \\
1950 & 2 & $?$ & $?$ \\
1954 & 3 & $?$ & $?$ \\
1955 & 9 & $?$ & 0,435 \\
1960 & 83 & 300 & 4,8 \\
1965 & 120 & 678 & 15,7 \\
1969 & 134 & 1089 & 30,7 \\
1979 & 121 & 2400 & 64,0 \\
1980 & 118 & 3447 & 75,0 \\
1981 & - & 4150 & - \\
\hline
\end{tabular}

\section{Funktion und Struktur der Massenmedien}

Lenins Funktionsbestimmung für die Presse (kollektiver Propagandist, kollektiver Agitator, kollektiver Organisator) gilt für alle Massenmedien der UdSSR. Für die Information in den Massenmedien gilt die leninsche Formel "Information ist Agitation durch Fakten". Auf Lenin gehen auch die Prinzipien für die Arbeit der Massenmedien zurück. Zumeist werden folgende genannt: Parteilichkeit, Wahrheit, Volksverbundenheit und Massencharakter. An erster Stelle steht die Parteilichkeit. Die Massenmedien müssen Klassencharakter haben, müssen parteilich sein. Die verschiedenen Bezeichnungen für die sowjetischen Massenmedien im Laufe ihrer Entwicklung zeigen dies: "Waffen“, „Transmissionsriemen“, „Stoßkräfte an der ideologischen Front", „Mittel der Masseninformation und Massenpropaganda" (so lautet die übliche Ubersetzung von "Massenmedien"). Die sowjetischen Massenmedien sind Teil eines umfassenden Erziehungs- und Lenksystems in der Hand der Parteiführung.

Artikel 50 der sowjetischen Verfassung vom Jahre 1977 sagt: „In Ubereinstimmung mit den Interessen des Volkes und zur Festigung und Entwicklung der sozialistischen Ordnung wird den Bürgern der UdSSR die Redefreiheit, die Pressefreiheit, Versammlungs- und Kundgebungsfreiheit, die Freiheit zur Durchführung von Straßenumzügen und Demonstrationen garantiert. Die Verwirklichung dieser politischen Freiheiten wird von den Werktätigen und ihren Organisationen durch die Bereitstellung öffentlicher Gebäude, Straßen und Plätze sowie durch die umfassende Informationsverbreitung und die Möglichkeit der Nutzung von Presse, Fernsehen und Rundfunk garantiert.“ Artikel 49 sichert ferner die Freiheit der Kritik zu. Ungeachtet dessen existiert und arbeitet ein Netz von Zensurbehörden, die darüber wachen, daß die Pressefreiheit den "Interessen des Volkes“ und der „Festigung und Entwicklung der sozialistischen Ordnung“ dient.

Die zentrale Lenkung wird vom Politbüro ausgeübt, das sich hierzu der Abteilungen des ZK bedient. Die ausführenden Institutionen sind das Staatskomitee für die Nachrichtenagentur $T A S S$, das Staatskomitee für das Druckwesen, das Staatskomitee für Fernsehen und Rundfunk, der sowjet ische Journalistenverband. (Man könnte auch noch den sowjetischen Schriftstellerverband hinzufügen.) Da die Presse sich in der Hand von Institutionen und Organisationen befindet, sind diese ebenso an der Verwirklichung der Weisungen der Parteispitze beteiligt (also z.B. der Komsomol, die Gewerkschaften). Die Vorsitzenden der 
genannten Staatskomitees haben Ministerrang, sie sind gleichzeitig hohe Parteifunktionäre. An der Spitze der Verbände, Institutionen und Organisationen stehen hohe Parteifunktionäre. Aufgabe der Massenmedien ist es, mitzuwirken an: 1. der Ausführung der von oben gegebenen Weisungen (also z.B. Erfüllung des Fünfjahresplans); 2. der Erziehung des „neuen Menschen“; 3. der Annahme des Marxismus-Leninismus in der jeweiligen von oben vorgenommenen Auslegung; 4. der Anerkennung der Führungsrolle der Partei, bzw. der jeweils führenden Parteispitze.

Die Staatsagentur TASS hat in der Sowjetunion das Nachrichtenmonopol, auch wenn einige Rundfunk- und Fernsehanstalten, einige große Zeitungen eigene Auslandskorrespondenten haben (so z. B. „Prawda“, „Isvestija“, „Trud“). An der Spitze von TASS steht ein von der Regierung ernannter Generaldirektor. TASS propagiert und informiert. TASSErklärungen sind offizielle Äu ßerungen. Die Nachrichtenauswahl erfolgt nach „marxistischleninistischer Analyse". In der UdSSR arbeiten 14 Republiksagenturen unter der Leitung von TASS. In der UdSSR umfaßt die beiderseitige Kabel-Fernschreibverbindung rund 300 Orte. Rund 4000 sowjetische Redaktionen erhalten das Material von TASS. Die sowjetischen Massenmedien sind auf das Angebot von TASS angewiesen und verpflichtet, sie dürfen z.B. nicht eigenmächtig die Nachricht eines ausländischen Rundfunksenders veröffentlichen. TASS fertigt ferner vertrauliche Sonderdienste an, die nicht veröffentlicht werden dürfen. Im Ausland hat TASS Büros und Korrespondentenpunkte in 126 Ländern. Mehr als 400 ausländische Agenturen, Informationsministerien, Redaktionen in 93 Ländern beziehen sich $T A S S$-Material in russischer, englischer, französischer, deutscher, spanischer oder arabischer Sprache. Mit rund 50 ausländischen Agenturen bestehen Verträge. TASS gehört zu den Weltagenturen.

Die sowjetische Nachrichtenagentur NOVOSTI (APN) wurde 1961 als „gesellschaftliche Agentur" vom sowjetischen Journalistenverband, vom sowjetischen Schriftstellerverband, von der sowjetischen Gesellschaft der Freundschaftsverbände zum Ausland und von der Gesellschaft zur Verbreitung politischer und wissenschaftlicher Kenntnisse gegründet. Ungeachtet dessen wird NOVOSTI vom ZK gelenkt, an der Spitze der Agentur steht ein hoher Parteifunktionär. Die Agentur dient der Auslandspropaganda der Sowjetunion. NOVOSTI hat im Ausland und in der UdSSR mehr als 140 Büros und Korrespondentenpunkte. Das in der UdSSR angebotene NOVOSTI-Material ist außerordentlich gering, während im Ausland Hunderte von Zeitschriften, Broschüren und Büchern in Millionenauflage in 26 Sprachen herausgebracht werden. Eine besondere Bedeutung hat der in mehreren Sprachen erscheinende „Digest der sowjetischen Presse, Sputnik".

Die sowjetische Presse gilt in der UdSSR immer noch als das wichtigste Massenmedium. obwohl inzwischen das Fernsehen erhebliche Konkurrenz macht. Am 5. Mai wird alljährlich der Tag der Presse begangen. Am 5.5.1912 erschien zum erstenmal legal die PRAWDA. Alle Zeitungen und Zeitschriften werden von Institutionen des Staates oder der Partei, von politischen oder gesellschaftlichen Organisationen herausgebracht. Anzeigen und Anzeigengeschäft spielen keine Rolle. Die vom ZK ausgehende Lenkung der Presse wird durch Institutionen des Staatskomitees, der einzelnen Organisationen und der Parteiorganisationen auf den verschiedenen Ebenen umgesetzt.

1981 erschienen in der UdSSR 8172 Zeitungen. Die folgende Tabelle zeigt die geographischadministrative Gliederung der sowjetischen Zeitungspresse im Jahre 1981. Die Sowjetunion gliedert sich in 15 Republiken. Diese Gliederung ist gleichzeitig eine Hierarchie, an deren Spitze die Allunionszeitungen stehen; an der Spitze der Allunionszeitungen wiederum steht die „Prawda“. Die Tabelle gibt keinen Aufschluß über die Wandzeitungen, die in Betrieben, Schulen, Kolchosen, militärischen Einheiten, Institutionen usw. ausgehängt werden. 


\begin{tabular}{|c|c|c|c|c|}
\hline $\begin{array}{l}\text { Zeitungen } \\
\text { der }\end{array}$ & $\begin{array}{c}\text { Anzahl der } \\
\text { Titel }\end{array}$ & $\begin{array}{l}\text { Einmalige } \\
\text { Auflage in } \\
1000 \text { Ex. }\end{array}$ & $\begin{array}{c}\text { Jahres- } \\
\text { gesamtauflage in } \\
1000 \mathrm{Ex} .\end{array}$ & $\begin{array}{l}\text { Durchschnitts- } \\
\text { auflage einer } \\
\text { Zeitung in } \\
1000 \text { Ex. }\end{array}$ \\
\hline Sowjetunion & $\begin{array}{c}31 \\
0.4 \%\end{array}$ & $\begin{array}{l}82.047 \\
46.0 \%\end{array}$ & $\begin{array}{c}21.344 .038 \\
52,5 \%\end{array}$ & $2.646,7$ \\
\hline Republiken & $\begin{array}{l}160 \\
2,0 \%\end{array}$ & $\begin{array}{l}25.964 \\
14.6 \%\end{array}$ & $\begin{array}{c}4.884 .435 \\
12.0 \%\end{array}$ & 162,3 \\
\hline $\begin{array}{l}\text { Regionen, } \\
\text { Gebiete, } \\
\text { Bezirke }\end{array}$ & $\begin{array}{l}329 \\
4.0 \%\end{array}$ & $\begin{array}{l}24.962 \\
14.0 \%\end{array}$ & $\begin{array}{l}6.395 .917 \\
15.8 \%\end{array}$ & 75,9 \\
\hline $\begin{array}{l}\text { Autonomen, } \\
\text { Republiken } \\
\text { od. Gebiete }\end{array}$ & $\begin{array}{l}97 \\
1.2 \%\end{array}$ & $\begin{array}{l}4.157 \\
2.3 \%\end{array}$ & $\begin{array}{c}1.020 .488 \\
2.5 \%\end{array}$ & 42,9 \\
\hline Städte & $\begin{array}{l}710 \\
8.7 \%\end{array}$ & $\begin{array}{l}17.321 \\
9.7 \%\end{array}$ & $\begin{array}{c}3.821 .943 \\
9.4 \%\end{array}$ & 24,4 \\
\hline Rayone & $\begin{array}{c}3004 \\
36.7 \%\end{array}$ & $\begin{array}{l}17.777 \\
10.0 \%\end{array}$ & $\begin{array}{c}2.805 .466 \\
\quad 6.9 \%\end{array}$ & 5,9 \\
\hline Betriebe & $\begin{array}{c}3231 \\
39.5 \%\end{array}$ & $\begin{array}{l}5.617 \\
3,1 \%\end{array}$ & $\begin{array}{l}399.530 \\
0,9 \%\end{array}$ & $\mathrm{I}, 7$ \\
\hline Kolchosen & $\begin{array}{l}610 \\
7.5 \%\end{array}$ & $\begin{array}{l}493 \\
0.3 \%\end{array}$ & $\begin{array}{l}11.316 \\
0,03 \%\end{array}$ & 0,8 \\
\hline Gesamt & $\begin{array}{l}8172 \\
100 \%\end{array}$ & $\begin{array}{c}178.338 \\
100 \%\end{array}$ & $\begin{array}{c}40.683 .133 \\
100 \%\end{array}$ & 21,8 \\
\hline
\end{tabular}

Aus dieser Tabelle geht hervor, daß die 31 Allunionszeitungen 52\% der Jahresgesamtauflage aller Zeitungen für sich beanspruchen können. Die täglich erscheinende „Prawda“ hat z.B. eine Auflage von 10.7 Millionen. Von allen in der UdSSR erscheinenden Zeitungen kommen 204 mit einer Auflage von 84,4 Millionen $(=47,3 \%)$ und einer Jahresgesamtauflage von 21.907 Millionen $(=53,8 \%)$ in Moskau heraus.

2.2 Erscheinungsweise der Zeitungen (1981)

$$
\begin{array}{ccc}
\text { Anzahl der } & \text { Einmalige } & \text { Jahresgesamt- } \\
\text { Titel } & \begin{array}{c}
\text { Auflage in } \\
\text { Auflage in } \\
1000 \text { Exemplaren }
\end{array} & 1000 \text { Exempl. }
\end{array}
$$

\begin{tabular}{lccc}
\hline Fünf- bis siebenmal & 468 & 107.310 & 31.923 .287 \\
in der Woche & $5.7 \%$ & $60,2 \%$ & $78.5 \%$ \\
Ein- bis viermal & 6.576 & 69.754 & 8.727 .389 \\
in der Woche & $80.5 \%$ & $39.1 \%$ & $21.4 \%$ \\
weniger als einmal & 1.128 & 1.274 & 32.457 \\
in der Woche & $13,8 \%$ & $0.7 \%$ & $0.1 \%$ \\
\hline Gesamt & & 178.338 & 40.683 .133 \\
& 8.172 & $100 \%$ & $100 \%$
\end{tabular}


Von den 8.172 Zeitungen erschienen 8.151 in 55 Sprachen der Völker der Sowjetunion, 21 in 10 Auslandssprachen. Von den 8.151 Zeitungen in Sprachen der Völker der Sowjetunion erschienen $5.271(=64,7 \%)$ in russischer Sprache, von der einmaligen Auflage entfallen auf die russischen Zeitungen $77,8 \%$, von der Jahresgesamtauflage $81,8 \%$.

Die sowjetische Zeitungspresse gliedert sich ferner auf nach der Thematik, bzw. der Organisation oder Institution, die für einen bestimmten Leserkreis zuständig ist. Durchweg gibt es bei dieser Gliederung ein Leitorgan in der Gruppe der Allunionszeitungen. Für die Zeitungen der Partei ist es die „Prawda“, für die der Regierung und der Sowjets die „Iswestija“, für die der Gewerkschaft „Trud“, für die der staatlichen Jugendorganisation „Komsomolskaja Prawda“ und „Pionerskaja Prawda“, für die der Streitkräfte „Krasnaja Swesda“, für die der Landwirtschaft „Selskaja Schisn“ usw. Die folgende Tabelle gibt Aufschluß darüber, wie stark einige dieser speziellen Zeitungsgruppen unter den Republiksund Gebietszeitungen vertreten sind. Ein Gesamtüberblick ist aufgrund fehlender Angaben nicht möglich.

Spezielle Zentralzeitungen der Republiken und Gebiete (198I)

\begin{tabular}{lccc}
\hline Zeitung & $\begin{array}{c}\text { Anzahl der } \\
\text { der Titel }\end{array}$ & $\begin{array}{c}\text { Einmalige } \\
\text { Auflage in } \\
\text { 1000 Exempl. }\end{array}$ & $\begin{array}{c}\text { Jahresgesamt- } \\
\text { auflage in } \\
\text { 1000 Exempl. }\end{array}$ \\
\hline Für Kinder und Jugendliche & 160 & 36.947 & 6.622 .476 \\
Für die Landwirtschaft & 9 & 12.071 & 3.537 .248 \\
Für Sport & 15 & 6.274 & 1.628 .700 \\
Für Industrie und Bauwesen & 9 & 3.198 & 630.701 \\
Für Transportwesen & 50 & 1.410 & 302.979 \\
Für Lehrer & 17 & 2.170 & 270.064 \\
Für Kunst und Kultur & 17 & 4.094 & 245.756 \\
\hline
\end{tabular}

Die Besonderheit der Zeitschrift läßt eine gleiche geographisch-administrative Hierarchie nicht zu, doch fällt eindeutig den politischen Zeitschriften die Leitfunktion zu. Die wichtigsten erscheinen in Moskau, an ihrer Spitze der „Kommunist“(früher Bolschewik) als ZK-Organ. Die sowjetischen Statistiken gliedern das Zeitschriftenwesen in: Journale (Zurnaly = Magazine, Illustrierte, Unterhaltungszeitschriften, kulturelle Zeitschriften usw.), die „Roman-Zeitung“ (früher wurde sie zu den wissenschaftlichen Zeitschriften gezählt), die „Notizen für den Agitator" (Bloknoty agitatora = spezielle Zeitschriften für Propagandisten, Agitatoren, Politinformatoren), Fachorgane (Sborniki = Zeitschriften, die sich mit einer speziellen Thematik befassen), Bulletins. Ein Vergleich mit im Westen üblichen Statistiken ist kaum möglich. Zu den sowjetischen Journalen gehören z. B. sowohl der „Kommunist“ wie die Illustrierte „Ogonjok“ wie die satirischen Zeitschriften. 


Zeitschriftenart

Anzahl der

Titel
Jahresgesamt- Durchschnitts-

Auflage in Auflage der

1000 Exempl. Zeitschriften in 1000 Exempl.

\begin{tabular}{lrrc}
\hline Journale & 1.457 & 2.370 .436 & 149,8 \\
Roman-Zeitung & 1 & 60.960 & $2.540,0$ \\
Notizen für den Agitator & 76 & 30.126 & 17,0 \\
Fachorgane & 170 & 9.861 & 16,7 \\
Bulletins & 3.491 & 644.655 & 20,0 \\
\hline Gesamt & & & \\
\hline
\end{tabular}

Das Schwergewicht liegt eindeutig bei den Journalen, die höchste Auflage hat das Journal „Rabotnica“ (Die Arbeiterin) mit 13 Millionen jeder Ausgabe. Von den 5.195 Zeitschriften erschienen $3.504(=67,4 \%)$ mit einer Jahresgesamtauflage von 2.212.204 Millionen Exemplaren $(=71 \%)$ in Moskau. Von den 5.195 Zeitschriften erschienen (1981) 4.921 in 46 Sprachen der Völker der UdSSR, 274 in 23 Auslandssprachen. Von den 4.921 in 46 Sprachen der Völker der UdSSR, 274 in 23 Auslandssprachen. Von den 4.921 Zeitschriften in Sprachen der Völker der UdSSR erschienen $4.195(=85,2 \%)$ in russischer Sprache; von der Jahresgesamtauflage entfallen auf die russischen Zeitschriften $80 \%$.

a. Erscheinungsweise der Zeitschriften (1981)

\begin{tabular}{lccc}
\hline & $\begin{array}{c}\text { Anzahl der } \\
\text { Titel }\end{array}$ & $\begin{array}{c}\text { Einmalige } \\
\text { Auflage in } \\
1000 \text { Exempl. }\end{array}$ & $\begin{array}{c}\text { Jahresgesamt- } \\
\text { auflage in } \\
\text { 1000 Exempl. }\end{array}$ \\
\hline Wöchentlich & 64 & 11.915 & 619.751 \\
Ein- bis viermal im Monat & 2.139 & $6,1 \%$ & $19,9 \%$ \\
weniger als einmal im Monat & $41,2 \%$ & 164.012 & 2.385 .994 \\
& 2.992 & $84,3 \%$ & $76,6 \%$ \\
Gesamt & $57,6 \%$ & 18.718 & 110.293 \\
& 5.195 & $9,6 \%$ & $3.5 \%$ \\
\hline
\end{tabular}

Das Schwergewicht liegt bei den Zeitschriften, die einmal im Monat erscheinen; es sind insgesamt 1.815 mit einer Jahresgesamtauflage von 1.619,4 Millionen Exemplaren. Über den Inhalt der Zeitschriften läßt sich - wegen der Verschiedenartigkeit - keine verallgemeinernde Aussage machen. Sex und Porno gibt es in sowjetischen Zeitschriften nicht. Eine besondere Bedeutung haben die literarischen Zeitschriften. Unter den atheistischen Zeitschriften ist "Nauka i religia“ (Wissenschaft und Religion) die wichtigste. 
b) Thematik von Zeitschriften in Auswahl (1981) in Prozentangaben von der Gesamtsumme der Zeitschriften

\begin{tabular}{lccc}
\hline Zeitschrift & $\begin{array}{c}\text { Anzahl der } \\
\text { Titel }\end{array}$ & $\begin{array}{c}\text { Einmalige } \\
\text { Auflage in } \\
1000 \text { Exempl. }\end{array}$ & $\begin{array}{c}\text { Jahresgesamt- } \\
\text { auflage in } \\
1000 \text { Exempl. }\end{array}$ \\
\hline Für Literatur & 221 & 62.420 & 1.121 .406 \\
Für Politik und Sozialökonomie & $4,2 \%$ & $32,0 \%$ & $35.9 \%$ \\
Für Kinder und Jugendliche & 787 & 67.074 & 966.557 \\
Für Technik, Industrie, & $15,1 \%$ & $34,4 \%$ & $31,0 \%$ \\
Transport usw. & $1,7 \%$ & 49.743 & 619.293 \\
& 1.982 & $25.5 \%$ & $19.9 \%$ \\
Für Gesundheit und Medizin & $38,1 \%$ & 26.150 & 488.882 \\
& 208 & $13,4 \%$ & $15,7 \%$ \\
Für Naturwissenschaft & $4,0 \%$ & 5.638 & 63.074 \\
und Mathematik & 465 & $2.9 \%$ & $2.0 \%$ \\
Für Sport & $8,9 \%$ & 4.202 & 47.135 \\
& 54 & $2,1 \%$ & $1,5 \%$ \\
& $1 \%$ & 2.391 & 28.339 \\
\hline
\end{tabular}

Ähnlich wie bei der Zeitungspresse fällt der hohe Anteil von Zeitschriften für Kinder und Jugendliche auf. Eine besondere Rolle spielen die literarischen Zeitschriften (in der UdSSR werden sie oft die „dicken Journale“ genannt). Ihre Tradition geht bis in die Zarenzeit zurück. In der Zeit Chruschtschows spielten sie bei der Entstalinisierung eine wichtige Rolle. Die in ihnen abgedruckten Erzählungen und Romane deuten vielfach politische und gesellschaftliche Probleme und Entwicklungstendenzen an.

Hörfunk und Fernsehen kommen in der Sowjetunion eine besondere Bedeutung zu. Die Bedeutung des Rundfunks wurde früh von Lenin erkannt. Mit Hilfe „der Zeitung ohne Papier und Entfernungen" (Lenin) konnten nicht nur schnell Entfernungen überwunden werden, man konnte auch die Analphabeten ansprechen und die Staatsgrenze überschreiten. Der 7. Mai wird in der UdSSR alljährlich als Tag des Rundfunks und des Fernsehens begangen. (Am 7.5.1895 hatte Prof. A. Popov in St. Petersburg eine Vorrichtung zur drahtlosen Ubertragung elektrischer Signale vorgeführt). Am 23.11.1924 begann Radio Moskau mit regelmäßigen Sendungen für das Inland, im Oktober 1929 wurde mit regelmäßigen Sendungen für das Ausland begonnen. Die Entwicklung begünstigte die Schaffung von Radioknoten (Zentrale Empfangsstellen, die das Programm an die Drahtempfänger weiterleiten). Auch heute überwiegt in der UdSSR die Anzahl der Drahtfunkempfänger gegenüber der Zahl der Ätherempfänger (Empfänger, mit deren Hilfe der Hörer sich selber das Programm im Äther sucht).

Auch der Hörfunk wird vom Politbüro über das ZK gelenkt. Zensur wird ausgeübt, unerwünschte Sendungen aus dem Ausland werden gestört. Vom ZK gehen Weisungen an das entsprechende Staatskomitee, das im Juli 1970 zum „Unionsrepublikanischen Staatskomitee für Fernsehen und Rundfunk" angehoben wurde. Ihm sind die Komitees, Studios usw. in den Republiken, Gebieten usw. untergeordnet, auch wenn sie Organe der örtlichen Sowjetmacht sind. Die Partei übt auf den verschiedenen Stufen Lenkung und Kontrolle aus. 
Die Zahl der Rundfunkstationen darf nicht verwechselt werden mit der Anzahl der Rundfunkkomitees, von denen Programme oder Programmteile hergestellt werden. Außer dem Moskauer Rundfunkkomitee gibt es in der UdSSR 162 Rundfunkkomitees. Der sowjetische Rundfunk besteht aus einer Kombination von Drahtfunk und hochfrequenter (drahtloser) Ubertragung. Das Drahtfunknetz ist über 2 Millionen km lang. Mit seiner Hilfe kann man das zentrale Programm mit dem lokalen Programm kombinieren. Die Zahl der Drahtfunkempfänger wird (1980) auf 74 Millionen, die der Ätherempfänger auf 70 Millionen geschätzt. Auf je 100 Familien entfallen 85 Rundfunkempfänger. Praktisch wird die gesamte Bevölkerung erreicht.

1980 strahlten die Programmsender zusammen täglich für die UdSSR 1312 Sendestunden in 68 Sprachen der Völker der UdSSR aus. Der bei weitem größte Teil der Sendungen wird in russischer Sprache ausgestrahlt. Für das Ausland strahlte die UdSSR täglich 240 Sendestunden in 77 Sprachen aus. Den Kern und Hauptteil des sowjetischen Rundfunkprogramms stellt in der UdSSR Moskau, d.h. die Programme von Republiksendern und örtlichen Sendern sind vielfach Ergänzung, Zusatz. Radio Moskau strahlt für die UdSSR täglich rund 178 Sendestunden aus.

Radio Moskau strahlt (1980) für die UdSSR faktisch 8 Programme aus. Das erste Programm (täglich 20 Stunden) enthält gesamtstaatliche und internationale Information, gesellschaftspolitische, künstlerische, unterhaltende Sendungen. Das zweite Programm (rund um die Uhr) nennt sich „Majak“ (Leuchtturm) und sendet Informationen und Musik. Das dritte Programm (täglich 16 Stunden) ist allgemeinbildend, literarisch-musikalisch ausgerichtet. Das vierte Programm (täglich 9 Stunden) sendet Musik, und zwar zum erheblichen Teil stereophon. Vom ersten Programm werden zeitversetzt vier Programme als Doubletten für die Hörer in Mittelasien, Sibirien, im Fernen Osten usw. ausgestrahlt. Ein fünftes Programm, das inhaltlich ähnlich wie das erste Programm gestaltet ist, wird für Sowjetbürger im Ausland ausgestrahlt (rund um die Uhr). Sowjetbürger hören täglich etwa anderthalb Stunden Rundfunk - vielfach auch bei der Ausübung anderer Beschäftigungen. Bevorzugt wird das Programm „Majak“. Millionen hören täglich - trotz der Störungen ausländische Sender, um jene Informationen zu erhalten, die von den sowjetischen Massenmedien nicht oder nur unvollständig verbreitet werden.

Seit Mitte der dreißiger Jahre werden in der UdSSR Fernsehsendungen mit Ton ausgestrahlt. In den fünfziger Jahren entwickelte sich das Fernsehen ziemlich unkoordiniert, ohne Verbindung der einzelnen Sender. Seit 1960 gilt das sowjetische Fernsehen als gleichberechtigtes Massenmedium neben Presse und Hörfunk. Von da an erfolgte die Entwicklung unter Anleitung und Kontrolle der Partei. In der zweiten Hälfte der sechziger Jahre wurden die „Fernsehinseln“ durch Umsetzer- und Kabelstrecken miteinander verbunden. Mit Hilfe von Umsetzer- und Kabelstrecken und von Satelliten (die ersten wurden 1965 gestartet) können inzwischen (1980) rund 86\% der sowjetischen Bevölkerung fernsehen bzw. das Moskauer Programm empfangen.

Die Lenkstruktur ist ganz ähnlich der des Rundfunks. An der Spitze steht das bereits erwähnte Staatskomitee, darunter die Komitees in den Republiken, autonomen Republiken usw. Die Anzahl der Fernsehzentren (Fernsehzentren produzieren Programme oder Programmteile) hat von 134 (1969) auf 1 I8 (1980) einschließlich der Fernsehzentrale Moskau abgenommen. Das Staatskomitee für Fernsehen und Rundfunk verfolgt konsequent eine Politik der Zentralisierung. Zugunsten des Zentralprogramms und der Republik-Programme wurden örtliche Studios geschlossen. Das System von Kabel- und Umsetzerstrecken, von Nachrichtensatelliten und Bodenempfangsstationen soll so ausgebaut werden, daß nicht nur die gesamte sowjetische Bevölkerung das erste Moskauer Programm sehen kann, sondern mehrere Moskauer Programme. Die Anzahl der Fernsehempfänger wird (1980) auf 75 Millionen geschätzt, davon 6,5 Millionen Farbfernseher. 64\% der sowjetischen Bevölkerung können zwei Programme empfangen, 
$14 \%$ können drei und mehr Programme empfangen. Auf je 100 Familien kommen 83 Fernsehempfänger. Ausländische Fernsehsendungen (aus Finnland, Polen usw.) können nur von einem kleinen Bruchteil der Bevölkerung empfangen werden. Mehr als 60 Telezentren produzieren Farbprogramme (1980).

Obwohl die Fernsehzentren eigene Programme herstellen, teilweise auch mehrere, ist das Moskauer erste Programm Kern aller Fernsehausstrahlungen. Alle Fernsehzentren zusammen strahlen täglich rund 2.000 Stunden Programm aus. Wieviele davon auf die verschiedenen Fernsehzentren entfallen, wieviele in russischer Sprache ausgestrahlt werden, geht aus den sowjetischen Quellen nicht hervor. Das Moskauer Zentralfernsehen produziert 10 Programme und sendet täglich mehr als 90 Stunden (1980). Das erste Programm (täglich 13,6 Stunden) umfaßt Information, gesellschaftspolitische, künstlerische und unterhaltende Sendungen. Das zweite Programm (täglich 4,5 Stunden) ist auf Moskau und Umgebung zugeschnitten. Das dritte Programm (täglich 7,1 Stunden) ist ein Unterrichts- und Bildungsprogramm. Das vierte Programm (täglich 6,4 Stunden) bietet Kunst, Unterhaltung, Sport. Sechs weitere Programme werden aus den erwähnten vier Programmen zusammengestellt und zeitversetzt über Boden- und Satellitenstrecken nach Mittelasien, Sibirien bis nach Kamtschatka ausgestrahlt. Das sowjetische Fernsehen ist Mitglied von INTERVIDENIE und INTERSPUTNIK. Das Fernsehen unterhält Kontakte zu mehr als 70 Ländern, der Rundfunk zu mehr als 90 Fernseh- und Rundfunkorganisationen im Ausland. Fernsehen und Rundfunk sind Mitglieder der OIRT.

Die Partei bezeichnet die sowjetischen Journalisten als „treue Helfer“. Journalisten werden an 24 Universitäten (1981) ausgebildet, insgesamt sind mehr als 14.000 Studenten in der Ausbildung. Der sowjetische Journalistenverband zählt mehr als 75.000 Mitglieder (1982). An seiner Spitze steht der Chefredakteur der Prawda, V. Afanasev. Die sowjetischen Journalisten werden bei ihrer Arbeit von den freiwilligen Arbeiter- und BauernKorrespondenten unterstützt. Etwa 6 Millionen solcher Korrespondenten liefern den Redaktionen Material. Vielfach handelt es sich um kritische Berichte. Auch die Millionen Leser- und Hörerbriefe, die in den Redaktionen von Presse, Rundfunk und Fernsehen eingehen, enthalten vielfach Kritik an Mißständen. Kritik wird gefordert und ist erwünscht. Kritik darf jedoch weder am Marxismus-Leninismus, noch an der Partei- und Staatsführung, noch an der sowjetischen Politik usw. geübt werden. Kritik muß aufbauend, muß parteilich sein.

\section{Probleme und Prognosen}

Die gegenwärtigen Probleme der Massenkommunikation in der UdSSR sind mitbedingt durch die Größe des Staates und die große Anzahl verschiedensprachiger Völkerschaften. Vor allem aber sind sie hervorgerufen durch das gesellschaftspolitische und wirtschaftliche System. Da in absehbarer Zeit keine grundlegende Änderung zu erwarten ist, ist bestenfalls mit der Lösung einiger technischer Probleme zu rechnen.

Als Stalin 1953 starb, war der Informationsfluß von unten nach oben teilweise zum Erliegen gekommen, teilweise total verzerrt. Ohne ausreichende Information von unten nach oben kann jedoch kein System auf Dauer funktionieren. Die Massenmedien genossen in der Bevölkerung nur noch geringe Glaubwürdigkeit. Die Sowjetbürger wußten, daß sie völlig unzureichend - selbst über die Vorgänge in der UdSSR - informiert wurden. Ohne wirksame Massenmedien muß jedoch ein System, das im Besitz des Meinungs- und Informationsmonopols ist, gelähmt werden. Schließlich war die UdSSR, als Stalin starb, vom Ausland weitgehend abgeschottet.

Die Nachfolger Stalins waren sich klar darüber, daß allein die Steigerung der Auflagen, die Ausweitung des Rundfunks, diese Probleme nicht lösen konnten. Die Meinungsforschung, die empirische Sozialforschung (sie waren unter Stalin eingestellt worden) wurden wieder ins 
Leben gerufen. Zwei Fragen interessieren die Machthaber besonders: Welche Meinungen gibt es in der sowjetischen Bevölkerung? - Welche Wirksamkeit haben Propaganda, Agitation, haben die Massenmedien?Seit der zweiten Hälfte der sechziger Jahre liegen in der UdSSR wissenschaftlich brauchbare Angaben aufgrund zahlreicher Untersuchungen vor. Obwohl nur ein kleiner Bruchteil dieser Untersuchungsergebnisse veröffentlicht wird, geht auch daraus hervor, daß die Wirksamkeit der Massenmedien den Wünschen der Machthaber nicht gerecht wird. Das verstorbene Staatsoberhaupt und Generalsekretär L. Breschnew hat dies sehr deutlich gesagt. Trotz der wiederbelebten Meinungsforschung sind auch heute noch die Machthaber nicht zufriedenstellend über die Meinungen, Haltungen und Vorgänge im eigenen Land informiert. Die Massenmedien sollten diesem Úbel vor allem durch Abdruck und Auswertung der Leser- und Hörerbriefe mit abhelfen. Grundsätzliche Kritik am System, am Marxismus-Leninismus, an der Partei, kann hierbei jedoch nicht zum Zuge kommen.

Die Wirksamkeit der Massenmedien wurde unter Chruschtschow u.a. dadurch gesteigert, $\mathrm{da} B$ die Massenmedien umfassender und lebendiger gestaltet wurden. Natürlich wurden Auflagen erhöht, Rundfunkprogramme erweitert usw. Man bemüht sich auch darum, das Nachrichtenangebot zu erweitern. Breschnew hat auch dies gefordert. Die sowjetische Führung ist hierin einem gewissen Druck ausgesetzt, da speziell Angehörige der "Intelligenzija“ Auslandssender hören. Dissidenten haben die Aufhebung der Zensur gefordert und nichtzensierte - meist maschinengeschriebene - Texte verbreitet. Durch Störung ausländischer Sender und die Einführung neuer Gesetze (Aburteilungen wegen "Verleumdung der UdSSR“) hat man zwar das Informationsmonopol der Herrschenden wieder abgesichert, kann jedoch mit Hilfe der Massenmedien das Informationsbedürfnis der Bevölkerung nicht befriedigen. Denn nach wie vor erfolgt Auswahl und Gestaltung der Informationen nach parteilichen Gesichtspunkten. Nach wie vor wird sorgfältig zensiert.

Alle Versuche zur Verbesserung der Wirksamkeit der Massenmedien erfolgten unter Anleitung und Kontrolle der Partei. Sie kümmert sich auch seit 1960 intensiv um das Fernsehen. So ist auch ein gewaltiges Netz von Zeitungen, Zeitschriften, Rundfunkstationen und Fernsehsendern entstanden. Die Vermehrung und Ausweitung der Massenmedien brachte jedoch neue Probleme mit sich. So hat man z.B. eine Reihe von Fernsehzentren wieder geschlossen, um die Zentralisierung wieder zu stärken. Die rund 30 Allunionszeitungen (also die zentralen Leitorgane) haben mehr als 50\% der Jahresgesamtauflage aller Zeitungen für sich.

Vor allem die Entwicklung des Fernsehens brachte Unruhe in das Mediengebäude. Im Durchschnitt widmet jeder Sowjetbürger dem Fernsehen täglich drei Stunden. Es gibt "Dauerglotzer", die Familie und gesellschaftliche Tätigkeit vernachlässigen. Besonders beliebt sind Unterhaltungssendungen, nicht etwa politische Sendungen. Man bemüht sich - unter Heranziehung von Wissenschaftlern - darum, Presse, Hörfunk und Fernsehen wie ein „Orchester“ einzusetzen und jedes Massenmedium aufgrund seiner spezifischen Sonderheiten zu nutzen. Hier wird es sicher Verbesserungen geben, doch laufen sie alle auf eine Verstärkung der politischen Wirksamkeit hinaus. Der Normalbürger, der sich abends vor seinen Fernseher setzt, möchte dann jedoch zumeist unterhalten und nicht belehrt werden.

Bis heute leidet die sowjetische Presse an Papiermangel. Auch fehlen hochqualifizierte Druckereien für guten Farbdruck in Zeitschriften. Schließlich wird ständig darüber geklagt, daß die Post nicht in der Lage ist, die Periodika rechtzeitig auszuliefern. Einige Allunionszeitungen werden daher - nach der Moskauer Vorlage - in verschiedenen Städten der UdSSR gedruckt (die Prawda z.B. wird in 47 sowjetischen Städten gedruckt). Der Papiermangel und die verspätete Zustellung von Periodika werden noch über Jahre hinweg nicht überwunden werden. Wann das Fernsehen - vor allem mit Hilfe von Satelliten - alle Bewohner der UdSSR erreichen wird, läßt sich schwer voraussagen. Ebensowenig läßt sich 
voraussagen, wann alle Sowjetbürger Farbfernseher in ihrer Wohnung stehen haben werden. Das Kassettenfernsehen steckt noch in den Kinderschuhen. Die gesamte Entwicklung des Fernsehens ist ja sowohl ein technisches wie ein finanzielles Problem.

Uber die Finanzierung der Massenmedien in der UdSSR wissen wir so gut wie nichts. Periodika sind außerordentlich billig. Anzeigen gibt es nur in wenigen Zeitungen. Man muß also davon ausgehen, daß Presse, Rundfunk und Fernsehen von Organisationen und Institutionen mitfinanziert werden. Für Rundfunk und Fernsehen gibt es keine Abonnementsgebühren, sondern nur einen Betrag beim Kauf des Gerätes. Von diesem Betrag kann das aufwendige Rundfunk- und Fernsehsystem sicher nicht leben. Die finanzielle Problematik wird allerdings in den sowjetischen Medien nicht diskutiert. Bei allgemein steigenden Kosten werden wohl Partei, Staat und die gesellschaftlichen Organisatoren mehr Geld für die Massenmedien aufbringen müssen.

Alle Veränderungen und Fortentwicklungen im sowjetischen Mediensystem (Erhöhung von Auflagen, Schaffung neuer Programme, Einsatz von Satelliten usw.) haben an der politischen Funktion der sowjetischen Massenmedien nichts geändert. Sie sind und bleiben von Partei und Staat gelenkte und kontrollierte Organe. Somit wird auch die Zensur fortbestehen. Uber die Verbesserung der Massenmedien wird in der UdSSR auch in der Offentlichkeit diskutiert. Ein Problem darf allerdings seit den zwanziger Jahren nicht öffentlich diskutiert werden: Informations-, Meinungs- und Pressefreiheit unterschiedlicher weltanschaulicher oder politischer Gruppen. Die Machthaber verweisen auf Artikel 50 der sowjetischen Verfassung und sagen, es gebe nirgendwo eine solche echte Meinungs- und Pressefreiheit auf der Welt wie in der UdSSR, weil in der UdSSR das Volk selber darüber bestimme.

\section{Literaturhinweise}

Es gibt keine sowjetische wissenschaftliche Veröffentlichung, die einen Gesamtüberblick über die Entwicklung der Massenmedien in der UdSSR gibt Angaben, Daten und Zahlen sind in hunderten von Artikeln, Broschüren und Büchern zu finden. Die Entwicklungstendenzen sowie Zahlenangaben lassen sich in den drei Ausgaben der „Großen Sowjetenzyklopädie“" (1926-1947, 1950-1958, 1969-1978) sowie in den seit 1957 erscheinenden Jahresbänden finden. Statistische Angaben über die Presse findet man in den jedes Jahr in „Pècat' SSSR v 19.. godu“ (Moskau) erscheinenden Veröffentlichungen.

Uber die politische Funktion der Massenmedien in der UdSSR, die Lenkung durch die Partei, informieren vor allem die beiden Dokumentarbände: $O$ partijnoj i sovetskoj pečati, radiovešcanii i televidenii. Moskau 1972. - KPSS o sredstvach massovoj informacii i propagandy. Moskau 1979.

Uber die aktuelle Entwicklung informieren vor allem folgende drei Zeitschriften: Zurnalist, Televidenie i Radiovescanie, - Vestnik Moskovskogo Universiteta. Zurnalistika.

Die wichtigsten nichtsowjetischen Buchveröffentlichungen über die sowjetischen Massenmedien sind folgende (aufgeführt in der Aufeinanderfolge des Erscheinens): A. Just: Die Presse der Sowjetunion. Methoden diktatorischer Massenführung. Berlin 1931. - A. Inkeles: Public opinion in Soviet Russia. Cambridge 1951. (Auch in französischer Sprache). - B. Kalnins: Der sowjetische Propagandastaat. Stockholm 1956. - T. Kruglak: The two faces of TASS. Minneapolis 1962. - A. Buzek: Die kommunistische Presse. Frauenfeld 1965. - J. Markham: Voices of the Red Giants. Communications in Russia and China. Ames, Iowa/USA 1967. - M. Hopkins: Mass Media in the Soviet Union. New York 1970. - H. Koschwitz: Pressepolitik und Parteijournalismus in der UdSSR und der Volksrepublik China. Düsseldorf 1971. - G.D. Hollander: Soviet Political Indoctrination: development in mass media and propaganda since Stalin. New York, Washington, London 1972. - M. Dewhirst/R. Farrell (H rsg.): The Soviet Censorship. Metuchen 1973. - B. Paulu: Radio and Television Broadcasting in Eastern Europe. Minnesota 1974. - L. Révész: Recht und Willkür in der Sowjetpresse. Eine presserechtliche und pressepolitische Untersuchung. Freiburg/Schweiz. 1974. - M. Lisann: Broadcasting to the Soviet Union. International Politics and Radio. New York, Washington, London 1975. - Ch. Kunze: Journalismus in der Sowjetunion. Eine Untersuchung über Aufgaben und Funktionen sowjetischer Journalisten. München, New York 1978. - H. Koschwitz (Hrsg.): Massenkommunikation in der Sowjetunion UdSSR. Sowjetische Beiträge zur empirischen Soziologie der Journalistik. Freiburg. München 1979.. 
P. Roth: SOW-INFORM. Nachrichtenwesen und Informationspolitik der Sowjetunion. Düsseldorf 1980. - P. Lendvai: Der Medien-Krieg. Wie kommunistische Regierungen mit Nachrichten Politik machen. Frankfurt, Berlin, Wien, 1981. - E. Hutter: Die sowjetische Informationspolitik. Ideologische Grundlagen und Wirkungsweise der Massenmedien. Freiburg/Schweiz 1981, - E.P. Propper: Media and the Russian public. New York 1981. - P. Roth: Die kommandierte öffentliche Meinung. Sowjetische Medienpolitik. Stuttgart 1982.

\section{SUMMARY}

The Soviet media system was created by Lenin and is developed by Stalin. The successors did not alter basically this system. The mass media are media for mass information and mass propaganda. As such they are instruments in the hands of the Party for the direction and education of the citizens. The total number of newspapers, periodicals, radio and television stations is impressive. Press and radio reach practically every citizen, the TV about $80 \%$ of the population. The mass media are not only controlled but also directed and of course censored. The citizens are protected from foreign news. Despite interference they however can receive some foreign broadcasts. Religious publications have a very small circulation indeed, but are also censored.

\section{RESUME}

Le système des média soviétiques a été fondé par Lenin et organisé par Stalin. Leurs successeurs n'ont en principe rien changé dans sa structure. Les mass-média sont des instruments du pouvoir du Parti. servant à guider et éduquer les citoyens. Le nombre total des journaux, périodiques et des stations de la radiodiffusion et télévision est impressionnant. La presse et la radiodiffusion atteignent pratiquement tous les citoyens, la télévision environ $\mathbf{8 0}$ pourcent. Les mass-média ne sont non seulement surveillés, mais guidés et bien entendu censurés. Les citoyens sont „protégés“ des nouvelles de l'étranger, mais malgré les interférences radiophoniques quelques émissions peuvent être écoutées. 11 y a des publications religieuses à tirages ridicules et elles aussi sont censurées.

\section{RESUMEN}

El sistema de media en la Unión Sovietica fué creado por Lenin y desarrollado por Stalin. Sus sucesores no han modificado mayormente este sistema. Los medios masivos de comunicación son el medio para la información y propaganda destinadas a las masas. Como tales ellos son instrumentos en las manos del Partido para dirigir y educar a los ciudadanos. El número total de diarios informativos, periódicos, estaciones de radio y televisión es impresionante. La prensa y la radio llega prácticamente a cada ciudadano. mientras que la televisión es vista por casi $80 \%$ de la población. Los "mass media“ no solo están controlados sino que ta mbién dirigidos y por supuesto censurados. Los ciudadanos son protegidos de las noticias extranjeras. Sin embargo y a pesar de las interferencias, ellos pueden recibir algunas emisiones de radios extranjeras. Las publicationes religiosas tienen una prequeña circulación y son también censuradas. 